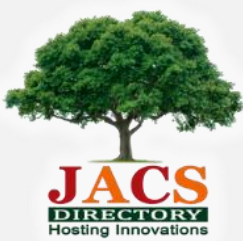

Journal of Nanoscience and Technology

Visit Journal at http://www.jacsdirectory.com/jnst

\title{
Photocatalytic and Antimicrobial Activities of Polyaniline Nanocomposites with Molybdates
}

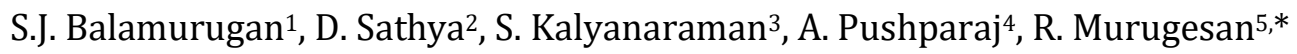 \\ Abishekapatti - 627 012, Tirunelveli, India. \\ ${ }^{2}$ Department of Physics, Sadakathullah Appa College, Palayamkottai, Tirunelveli- 627002 , Tamilnadu, India. \\ ${ }^{3}$ Department of Physics \& Principal (Rtd), Sri Paramakalyani College, Alwarkurichi - 627 412, Tamilnadu, India. \\ ${ }^{4}$ Department of Zoology, TDMNS College, T. Kallikulam, Tirunelveli - 627 113, Tamilnadu, India. \\ ${ }^{5}$ Department of Chemistry, TDMNS College, T. Kallikulam, Tirunelveli - 627 113, Tamilnadu, India.
}

${ }^{1}$ Research Scholar (Reg. No.: 8234), PG and Research Department of Physics, Sri Paramakalyani College affiliated to Manonmaniam Sundaranrar University,

\section{ART ICLE DETAILS}

Article history:

Received 20 March 2019

Accepted 29 April 2019

Available online 04 June 2019

Keywords:

Polyaniline

Nanocomposites

Photo-Catalytic Activity

Antimicrobial Activity

\begin{abstract}
A B S T R A C T
The present study describes the process for the formation of PANI nanocomposite incorporated with metal $(\mathrm{Cd}, \mathrm{Bi}, \mathrm{Ba})$ molybdates of binary metal oxide. The PANI and its nanocomposites were prepared by $0.1 \mathrm{M}$ aniline in $100 \mathrm{~mL}$ of $1 \mathrm{M}$ sulphuric acid in the presence or absence of $50 \mathrm{mg}$ of metal molybdate by chemical polymerization using $0.1 \mathrm{M}$ APS as oxidant in $100 \mathrm{~mL}$ of $1 \mathrm{M}$ sulphuric acid. The polymerisation yields are increased due to incorporation of metal molybdates. The growth of polymerization is also enhanced. The interaction of metal molybdates into PANI matrix leads to increase in very high conductivity, heavy interaction between the two-polymer chain lead to increase in stability of the polymer. In the incorporation of metal molybdate into the polymer matrix leads to increase in conductivity of about 5, 4 and 2 times with respect to the original sample (PANI) as in PANI-CdMoO ${ }_{4}$, $\mathrm{PANI}_{-}-\mathrm{BiMoO}_{4}$ and $\mathrm{PANI}-\mathrm{BaMoO}_{4}$ respectively. The incorporation of metal molybdates is confirmed by XRD and UV-VIS spectral studies. The XRD studies are also suggested the presence of chemical interaction between polymer chain and incorporated metal molybdates. The antimicrobial activity of the synthesized material PANI and PANI-nanocomposites were tested against four bacteria, Escherichia coli, Staphylococcus aureus, $P$. aeruginosa and Proteus vulgaris using disc diffusion method. The nanocomposite doped polymer possesses excellent antibacterial property. The results on MB dye degradation showed that PANI-SO ${ }_{4}^{2-} / \mathrm{CdMoO}_{4}$ photocatalyst, under visible light degraded $80.2 \%$ of $\mathrm{MB}$ dye after $180 \mathrm{~min}$. The photo-degradation study, thus, suggests that the light absorption of PANI and catalytic role of $\mathrm{CdMoO}_{4}$ could be coupled for high photo-catalytic functionality. This work opens a new avenue for the design of new modified photo-catalysts with high activity for the large-scale water treatment and other applications.
\end{abstract}

\section{Introduction}

Today the prospects in optical devices are closely related to highly efficient luminescent semiconductor nanomaterials, which possess unique electronic properties. Further progress in evolvement of luminescent materials are associated with enhancement of their stability, simplification of synthetic methods, as well as development of novel procedures for preparation of nanostructures with predefined optical properties. In the same time the possibility to accurately control any functional properties of nanomaterials is affected by three governing factors: the size, shape and dispersity of nanomaterials, which possess the number of structural defects and the uniformity of chemical composition. Among the mixed metal oxides, some metal molybdates are found to be very interesting due to their structural, electronic and catalytic properties. Calcium molybdate with a scheelite structure has been of practical interest because of their attractive luminescence property [1]. Cobalt and nickel molybdates are important components of industrial catalysts for the partial oxidation of hydrocarbons [2]. The crucial microstructure-based properties of these metal molybdates are likely to be influenced by the dimensional manipulation of their grain-sizes and the grain boundary effects in a polycrystalline material. This study is aimed as a simple method of preparation of nanomaterials, metal molybdate systems and its incorporation of polymer matrix. The important advantage of implementation of matrix techniques is the possibility to accurately control dispersion of nanomaterials.
The incorporation of metal molybdate nanomaterials into the conducting polymer offers enhanced performance for both the host and the guest [3].

The applications of these nanocomposites have also been extended to various fields such as, sensors [4, 5], photovoltaic cells [6], memory devices [7], and supercapacitors [8]. The preparation of nanomaterial implanted conducting polymer composites have been described by various methods, including template method for growing metal nanoparticles and polymers into nanostructures and incorporation of preformed nanoparticles during polymerization of monomers. The main challenge is identifying the right combination of organic and inorganic molecular building blocks that enhances the properties of each component while reducing their limitations. This challenge provides a unique opportunity for synthetic material scientist for developing new materials with synergetic behaviour leading to improved performance or completely new properties.

Organic-inorganic polymer nanocomposites have potential applications depending upon their synthetic techniques and the nature of the inorganic materials. However, among various conducting polymers, polyaniline and its nanocomposites have scarcely been investigated. Cadmium molybdate, Bismuth molybdate and Barium molybdate nanomaterials have special physico-chemical properties arising from the size effect and high specific surface area, which may be different from their atomic or bulk counterparts. 


\section{Experimental Methods}

\subsection{Materials}

All the chemicals used in this work were commercially available AR or GR grade. Aniline, ammonium perdisulphate, sodium molybdate were purchased from SD Fine Chemicals, India. cadmium nitrate, bismuth nitrate, barium nitrate was purchased from Qualigens fine chemicals, India. Aniline was distilled over zinc dust to remove all oxidized products formed during storage.

\subsection{Preparation of Metal Molybdate Powders}

The metal nitrates $(\mathrm{Cd}, \mathrm{Bi}$ and $\mathrm{Ba}$ ) were dissolved in deionised water. $25 \mathrm{~mL}$ of the metal nitrate solution $(0.01 \mathrm{M})$ was poured into an equal volume of aqueous solution of sodium molybdate $(0.01 \mathrm{M})$, to precipitate out the respective metal molybdate from the solution [9]. The respective metal molybdate were separately collected upon filtration and were thoroughly washed with water to make it free of sodium ions and checked quantitatively.

\subsection{Preparation of PANI and Its Nanocomposite with Metal Molybdates}

The PANI and its nanocomposites were prepared by $0.1 \mathrm{M}$ aniline in 100 $\mathrm{mL}$ of $1 \mathrm{M}$ sulphuric acid in the presence or absence of $50 \mathrm{mg}$ of metal molybdate by chemical polymerization using APS as oxidant in $100 \mathrm{~mL}$ of $1 \mathrm{M}$ sulphuric acid. The stirring of the reaction mixture was continued for half an hour. After addition of APS, polymerization was allowed to proceed further for overnight in a refrigerator. The green precipitate formed was filtered, washed with water and then ethanol and acetone until the filtrate became colorless and dried in an air oven at $80{ }^{\circ} \mathrm{C}$ for about 4 hours. The dried polymer samples were grinding into a fine powder and the yield were noted. The polymer samples were stored in air-proof sealed plastic covers.

\subsection{Characterization of Polymer Samples}

All the polymeric samples were characterized by DC electrical conductivities (four probe method) and UV-VIS spectral studies. All the polymeric materials are dissolved in NMP, which is a highly coordination solvent. UV-VIS spectra of the synthesized polymer samples were recorded at room temperature in NMP 390-900 $\mathrm{nm}$ range with UV-VIS spectrophotometer with computer software in matched $1 \mathrm{~cm}$ quartz cuvettes available at Department of PG Chemistry and Research Centre, Adithanar Arts and Science College, Tiruchendur, Tamil Nadu. XRD pattern for all the polymeric samples were recorded on JEOL (Japan) JDX-8030 computer-controlled X-ray diffractometer (CECRI, Karaikudi) with CuK $\alpha$ radiation $(\lambda=1.5420 \AA)$, scanning in the range of 3 to 80 in a step of 0.1 per sec.

\subsection{Studies of Antimicrobial Activity}

The antibacterial activity of polymer samples was investigated against Escherichia coli, Staphylococcus aureus, P. aeruginosa and Proteus vulgaris microorganisms by disk diffusion method in accordance with the procedure described by Hwang and Ma [10]. This method is a mean of measuring the effects of an antimicrobial agent on bacteria growth in a culture. Muller-Hinton Agar (MHA) powder was used as a culture medium for bacteria growth. To prepare the culture medium, $19 \mathrm{~g}$ of agar was dissolved into $500 \mathrm{~mL}$ of distilled water, and then a transparent brown solvent was achieved via boiling the solution. MHA medium (15 mL) was sterilized at $120{ }^{\circ} \mathrm{C}$ for about 1 hour in autoclave, cooled to room temperature and then poured into sterilized petri dishes $(10-90 \mathrm{~mm})$. After cooling over 24 hours, the bacteria are swabbed uniformly across the culture plate. Filter-paper disks were placed on the surface of agar. To evaluate the antibacterial activity, $40 \mu \mathrm{L}$ of each sample was dropped moderately on disks' surface using a sampler. All tests were done under laminar flow hood. Finally, all petri dishes containing bacteria and antibacterial reagents were incubated at $37{ }^{\circ} \mathrm{C}$ for $24 \mathrm{~h}$. At the end of incubation period, the diameters of inhibition zones formed around disks, were determined and presented in $\mathrm{mm}$. The size of inhibition zone measures the efficacy of sample. A more effective sample produces a larger clear area around the disk. The results concerning antibacterial activity were expressed as strong activity (>13 mm), moderate activity $(6-12 \mathrm{~mm})$, weak activity $(5 \mathrm{~mm}$ ) or no activity (inhibition zone $<5 \mathrm{~mm}$ ).

\subsection{Studies of Photocatalytic Activity}

The photodegradation studies were carried out and the light source was the visible light from $150 \mathrm{~W}$ tungsten lamp $(\lambda>380 \mathrm{~nm}$; irradiation time = $180 \mathrm{~min}$ ). The photoreactor wall was covered by aluminum foil during the course of reaction. The $\mathrm{pH}$ of the medium was adjusted using $0.1 \mathrm{M} \mathrm{HCl}$ or
$\mathrm{NaOH}$. Air flow at constant rate was made using air pump. The temperature and pressure of the photoreactor was maintained at $28 \pm 2{ }^{\circ} \mathrm{C}$ and atmospheric pressure. $100 \mathrm{~mL}$ MB dye solution was mixed thoroughly with required quantity of photocatalyst and shaken for 30 minutes to reach adsorption/desorption equilibrium in the absence of light. Photodegradation study of MB dye for the four catalysts PANI, PANI$\mathrm{CdMoO}_{4}, \mathrm{PANI}_{-} \mathrm{BiMoO}_{4}$ and PANI-BaMoO${ }_{4}$ was carried out in light, constant air flow. The first MB dye sample from dark equilibrium was taken to determine the initial concentration. After that tungsten lamp was switched on to start photocatalysis. Samples were taken periodically at various time intervals for $180 \mathrm{~min}, 2 \mathrm{~mL}$ a sample was drawn out for every $15 \mathrm{~min}$ intervals. The experimental samples were centrifuged and filtered through a Whatman filter paper No.1 to separate catalyst particles. The UV-Visible absorption spectra of the supernatant solution were analyzed using a UVVisible spectrometer in quartz cuvettes to monitor the characteristic absorption peak of MB at $650 \mathrm{~nm}$. Then the absorption of MB aqueous solution was measured by UV-Vis spectrophotometer. In order to evaluate the photocatalytic performance, the degradation rate (D) can be calculated using equation [11].

$$
\mathrm{D}=\frac{\mathrm{C}_{\mathrm{o}}-\mathrm{C}_{\mathrm{t}}}{\mathrm{C}_{\mathrm{o}}} X 100 \%
$$

where, $C_{o}$ is the initial concentration of $\mathrm{MB}(100 \mathrm{mg} / \mathrm{L})$; $\mathrm{C}_{\mathrm{t}}$ is the concentration after $t$ min of photocatalytic degradation.

\section{Results and Discussion}

\subsection{Polymerization Yield and DC Conductivity Measurements}

The chemical oxidative polymerization of aniline in the presence or absence of metal molybdate powder was carried out in aqueous sulphuric acid medium using APS as oxidizing agent. The preparation yield of PANI and its nanocomposites with metal molybdates are reported in the Table 1. The yield is enhanced due to the incorporation of metal molybdates in all the PANI samples, as compared with PANI sample except Barium molybdate. This retarded the polymerization and hence the yield is reduced. But in the case of $\mathrm{CdMoO}_{4}$ and $\mathrm{BiMoO}_{4}$ it is clearly indicated that the incorporation of metal molybdates enhanced the polymerization yield as well as the rate of reaction or the growth of polymerization chain. Many studies support the similar effect of polymerization of PANI.

Table 1 Polymerization yield and DC conductivity of PANI and its nanocomposites with metal molybdate

\begin{tabular}{lllll}
\hline Sample & Aniline & ADS & Yield (g) & $\sigma$ \\
\hline PANI & $0.10 \mathrm{M}$ & $0.10 \mathrm{M}$ & 2.643 & 0.6626 \\
PANI-CdMoO $_{4}$ & $0.10 \mathrm{M}$ & $0.10 \mathrm{M}$ & 2.741 & 2.5514 \\
PANI-BiMoO $_{4}$ & $0.10 \mathrm{M}$ & $0.10 \mathrm{M}$ & 2.741 & 2.0787 \\
PANI-BaMoO $_{4}$ & $0.10 \mathrm{M}$ & $0.10 \mathrm{M}$ & 1.672 & 1.0753 \\
\hline
\end{tabular}

Variation of conductivity value is due to the types of polymerization and by the nature of oxidant. It is noticeable from the data (Table 1) that the conductivity of PANI is less than that of composites. By the inclusion of molybdates enhances the conductivity of PANI. By the interaction of molybdates into PANI matrix lead to increase in very high conductivity, heavy interaction between the two-polymer chain lead to increase in stability of the polymer. In most cases, incorporation of metal molybdate into the polymer matrix lead to increase in conductivity of about 5,4 and 2 times with respect to the original sample as in $\mathrm{PANI}-\mathrm{CdMoO}$, PANI$\mathrm{BiMoO}_{4}$ and PANI-BaMoO 4 . By the incorporation of molybdate into the polymer matrix improve conductivity and enhance stability of the resulting molecule [12]. Electrical conductivity of the PANI materials is important in redox reactions. If the active substance, a binary metal oxide has two center: redox and acid-base, changes in conductivity of the polymer matrix, which affect mainly redox properties of the catalyst have an influence of selectivity of reaction over these catalysts.

\subsection{UV-Vis Spectral Studies}

All the PANI materials are dissolved in NMP, which is a highly coordination solvent. It can easily dissolve. Fig. 1 shows the UV-Vis absorption spectra of PANI samples. All the PANI samples are observed ( $\pi$ $\left.\pi^{*}\right)$ extended conjugation at around $350-385 \mathrm{~nm}$; $\left(n-\pi^{*}\right)$ polaronic peak around 530-630 $\mathrm{nm}$ and above $800 \mathrm{~nm}$ has aemeraldine base form in tail structure. All the PANI samples shape of graph, intensity peak of height and intensity of positions is different. It indicates that all polymer materials have different compositions. The solubility of composite materials is high due to high peak intensity. The intensity of the second 
band at 530-630 nm in the spectrum of the polymer prepared using metal molybdates are higher than the polymer prepared using APS alone, which indicates that the relative amount of the quinoiddiimine unit in the PANI nanocomposites prepared using metal molybdates are more than the polymer prepared using APS alone. This implies that the metal molybdates had also an effect on the conjugate structure of the conducting PANI. That indicates the presence of polarons resulting from the doping process [13]. A given polymer consist of polarons at one doping level and bipolarons at a different doping level or consist of significant amounts of polarons and bipolarons with each other under certain conditions. Based on the UV-Vis spectra it is clear that polymer chains have different electronic structures in NMP. This difference in electronic structures can be attributed only to their difference in conformational structures. The dispersion of the polaron band is, in particular, very sensitive to the geometric arrangement of polymer backbone because the polaron band is formed via overlap between polarons of adjacent tetrameric units. The polarons of individual tetrameric units are isolated from each other because of the twist defects between aromatic rings. Therefore, the polaron band has little dispersion in energy.

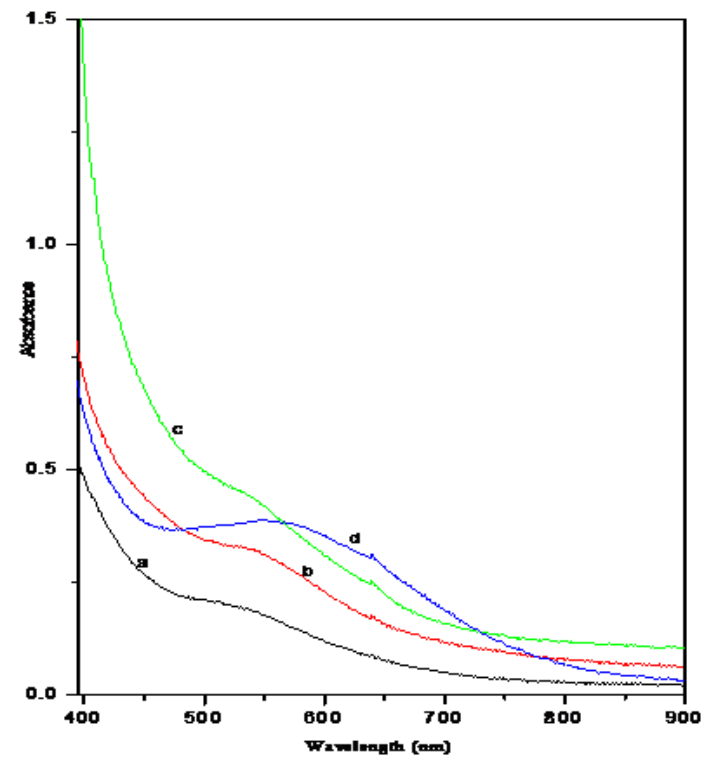

Fig. 1 UV-Visible spectra for a. PANI; b. PANI-CdMoO 4 ; c. PANI-BiMoO 4 ; and d. PANI$\mathrm{BaMoO}_{4}$ in NMP

\subsection{XRD Studies}

The crystal structures of the synthesized PANI and its nanocomposites of PANI-CdMoO 4 , PANI-BiMoO4, PANI-BaMoO 4 were investigated by powder XRD. Fig. 2(a-d) shows the XRD pattern of PANI, PANI-CdMoO $\mathrm{PANI}_{-} \mathrm{BiMoO}_{4}$, and PANI-BaMoO 4 respectively. The XRD pattern of pure PANI has a peak at 23.06 and 26.07 in Fig. 24(a) indicates its semicrystalline amorphous nature. This characteristic peak of PANI is ascribed to the periodicity in parallel and perpendicular directions of the polymer chain. In Fig. 2(b) the peaks observed for at $2 \theta$ angle at 19.41, 23.08, 25.41, $27.69,29.50,44.71,48.49$, and 54.13 confirms the formation of PANI$\mathrm{CdMoO}_{4}$ nanocomposites and it is crystalline in nature. However, the XRD

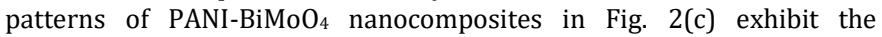
characteristic peak of PANI along with the crystalline peaks of $\mathrm{BiMoO}_{4}$, owing to the systematic alignment of polymer chain. The increase in ordering of polymer nanocomposites with the addition of $\mathrm{Cd}, \mathrm{BiMoO}_{4}$ nanoparticles indicates that the structure of PANI is strongly influenced by the nanoparticles. The increase in degree of regularity in arrangement or ordering of polymer chain is due to the strong interfacial interaction between the polymer and nanoparticles. Hence, the orientation of conducting PANI nanocomposites is of much interest, due to more highly ordered polymer matrix it could display a conductive property whereas $\mathrm{BaMoO}_{4}$ doped PANI samples were declaining effect of crystallinity which is observed as 18,10 and 25.69 respectively. It is obtained amorphous nature and low conductivity as compared to their two nanocomposites. The average size of the Metal molybdate doped PANI nanoparticles were calculated from the full width of half maximum (FWHM) values of the diffraction peaks using Debye-Scherrer formula (Table 2) using the DebyeScherrer formula , $d=0.94 \lambda / \beta \operatorname{Cos} \theta$, where $\lambda$ is the wavelength of $x$-ray used $(\lambda=1.542 \AA), \beta$ is the full width of diffraction line at half maximum intensity (FWHM) and $\theta$ is the Bragg's angle. The average particle sizes are reduced due to incorporation binary metal oxides of $\mathrm{Cd}$, $\mathrm{BiMoO}_{4}$. This observation is also supported with other characterisation of DC conductivity, and UV-Visible spectroscopy studies.

https://doi.org/10.30799/jnst.S11.19050304
Table 2 XRD data for PANI and its composites with molybdates

\begin{tabular}{llllll}
\hline Samples & $\begin{array}{l}\text { Position } \\
{\left[{ }^{\circ} \mathrm{Th} .\right]}\end{array}$ & $\begin{array}{l}\text { FWHM } \\
{\left[{ }^{\circ} 2 \mathrm{Th} .\right]}\end{array}$ & $\begin{array}{l}\text { d-spacing } \\
{[\AA]}\end{array}$ & $\begin{array}{l}\text { Particle } \\
\text { Size }[\mathrm{nm}]\end{array}$ & $\begin{array}{l}\text { Average } \\
\text { Particle Size }\end{array}$ \\
\hline PANI & 23.3341 & 0.2086 & 3.98721 & 38.89 & 43.345 \\
& 26.0782 & 0.1702 & 3.41420 & 47.8 & \\
PANI-CdMoO $_{4}$ & 19.4125 & 0.2085 & 4.78310 & 38.68 & \\
& 23.0843 & 0.2005 & 3.84978 & 40.4 & 38.280 \\
& 25.4175 & 0.18 .55 & 3.41853 & 43.90 & \\
& 27.6945 & 0.1470 & 3.12452 & 55.60 & \\
& 29.5050 & 0.4064 & 3.02500 & 20.0 & \\
& 44.7101 & 0.6237 & 2.02526 & 13.8 & \\
& 48.4918 & 0.1735 & 1.87352 & 45.80 & \\
PANI-BiMoO $_{4}$ & 54.3381 & 0.1856 & 1.72850 & 48.12 & \\
& 19.5521 & 1.5744 & 4.54033 & 05.1 & \\
& 23.3341 & 0.2086 & 3.98721 & 38.89 & 38.645 \\
& 26.3804 & 0.1590 & 3.37577 & 51.3 & \\
& 25.6353 & 0.1788 & 3.22177 & 45.57 & \\
& 29.7781 & 0.1813 & 2.99788 & 45.3 & \\
& 48.4918 & 0.1735 & 1.87352 & 45.80 & \\
& 23.3341 & 0.2086 & 3.98721 & 38.89 & 40.295 \\
PANI-BaMoO $_{4}$ & 25.6975 & 0.1953 & 3.96251 & 41.70 & \\
& & & & & \\
& & & &
\end{tabular}
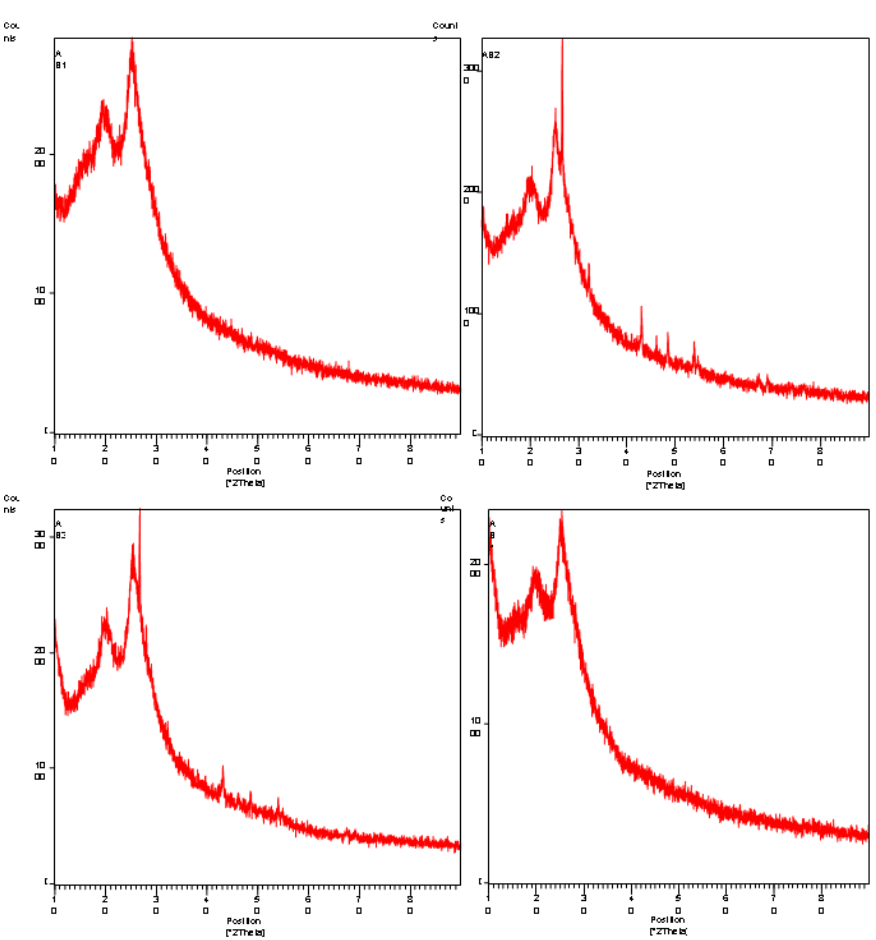

Fig. 2 XRD pattern for a. PANI; b. PANI-CdMoO 4 ; c. PANI-BiMoO 4 ; and d. PANI-BaMoO 4

\subsection{Studies of Antimicrobial Activities}

PANI and PANI nanocomposites with metal molybdates have been tested for its antimicrobial activity against Escherichia coli, Staphylococcus aureus, P. aeruginosa and Proteus vulgaris. The results of antimicrobial activity of PANI and PANI nanocomposites with metal molybdates assessed based on the average diameter of zones of inhibition, which confirms that PANI nanocomposites have an enhanced antimicrobial activity compared to PANI. PANI and PANI nanocomposites also show a potent antibacterial activity compared to antimicrobial activity of other materials in both solvent like m-cresol (Table 3 and Fig. 3) and NMP (Table 4 and Fig. 4). NMP is co-ordinate solvent and neutral and their antimicrobial activity is less as compared with other solvent of m-cresol. $\mathrm{m}$-Cresol is high acidic nature and more soluble in all PANI and its nancomposites [14]. The nanocomposite doped polymer possesses excellent antibacterial property.

Table 3 Anti-microbial activity data in m-cresol for PANI and its nanocomposites with molybdates

\begin{tabular}{lllllll}
\hline Microorganisms & $\begin{array}{l}\text { m-Cresol } \\
\text { (solvent) NC }\end{array}$ & $\begin{array}{l}\text { PANI } \\
\text { PANI- } \\
\mathrm{CdMoO}_{4}\end{array}$ & $\begin{array}{l}\text { PANI- } \\
\mathrm{BiMoO}_{4}\end{array}$ & $\begin{array}{l}\text { PANI- } \\
\mathrm{BaMoO}_{4}\end{array}$ & $\begin{array}{l}\text { Chloram } \\
\text { phenical PC }\end{array}$ \\
\hline Escherichia coli & 16 & 21 & 29 & 26 & 23 & 32 \\
$\begin{array}{l}\text { Staphylococcus } \\
\text { aureus }\end{array}$ & 17 & 19 & 27 & 25 & 22 & 33 \\
$\begin{array}{l}\text { P.aeruginosa } \\
\text { Proteus vulgaris }\end{array}$ & 15 & 19 & 25 & 23 & 19 & 24 \\
\hline
\end{tabular}




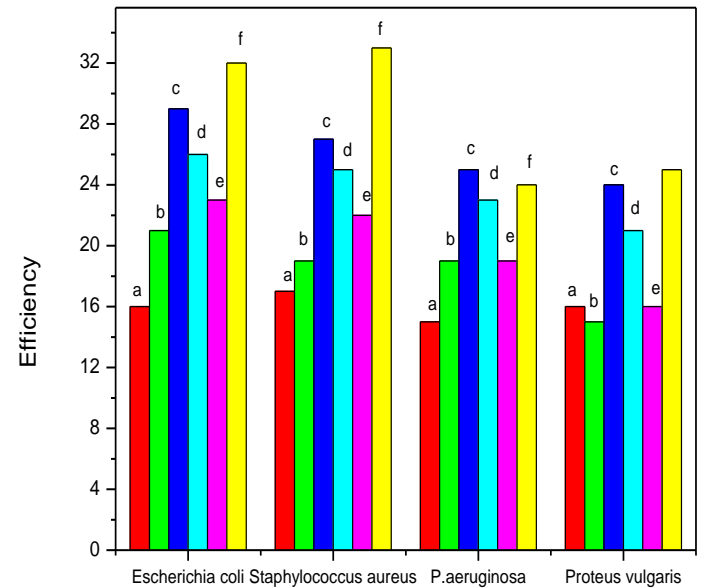

Fig. 3 Anti-microbial a. m-Cresol (solvent) NC; b. PANI; c. PANI-CdMoO ${ }_{4}$; d. PANI$\mathrm{BiMoO}_{4}$; e. PANI- $\mathrm{BaMoO}_{4}$; and f. Chloramphenical PC

Table 4 Anti-microbial Activity data in NMP for PANI and its nanocomposites with molybdates

\begin{tabular}{lllllll}
\hline Microorganisms & $\begin{array}{l}\text { NMP } \\
\text { (solvent) NC }\end{array}$ & $\begin{array}{l}\text { PANI } \\
\mathrm{PANI}^{2}\end{array}$ & $\begin{array}{l}\text { PANI- } \\
\mathrm{CdMoO}_{4}\end{array}$ & $\begin{array}{l}\mathrm{PANI}_{4} \\
\mathrm{BaMoO}_{4}\end{array}$ & $\begin{array}{l}\text { Chloram } \\
\text { phenical PC }\end{array}$ \\
\hline $\begin{array}{l}\text { Escherichia coli } \\
\text { Staphylococcus }\end{array}$ & 6 & 17 & 29 & 27 & 25 & 32 \\
$\begin{array}{l}\text { aureus } \\
\text { P.aeruginosa }\end{array}$ & 6 & 20 & 27 & 25 & 24 & 33 \\
Proteus vulgaris & 6 & 20 & 23 & 19 & 21 & 24 \\
\hline
\end{tabular}

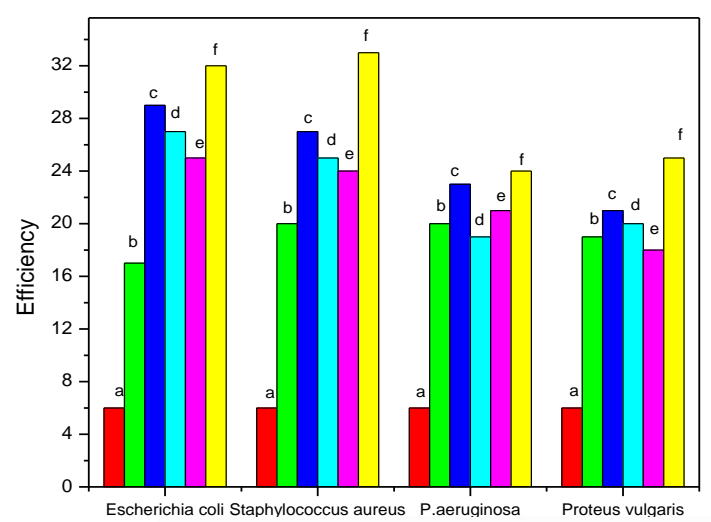

Fig. 4 Anti-microbial a. NMP(solvent) NC; b. PANI; c. PANI-CdMoO4; d. PANI-BiMoO4; e. PANI-BaMoO 4 ; and f. Chloramphenical PC

\subsection{Studies of Photodegradation}

The degradation of MB dye in the absence of catalyst indicated at blank condition no significant degradation is observed under light irradiation (3.1\% was achieved). The effect degradation of MB dye is studied shown in Table 5 and Fig. 5. The four catalysts PANI, PANI-CdMoO ${ }_{4}, \mathrm{PANI}_{-}-\mathrm{BiMoO}_{4}$ and PANI-BaMoO ${ }_{4}$ exhibited the MB dye decomposition of $25.2 \%, 55.0 \%$, $80.2 \%$ and $76.1 \%$ respectively. For heterogeneous catalysis particle size is more importance. The increase in particle size decreases the net available surface area of the catalyst, which in turn decreases the number of available active sites [15] for the reaction. By comparing other composite, PANI-CdMoO 4 has more stable photocatalytic activity toward degradation of MB dye in liquid-phase.

Table 5 Photocatalytic variation of types of catalyst

\begin{tabular}{llllll}
\hline Time (s) & Blank & PANI & $\begin{array}{l}\text { PANI- } \\
\mathrm{CdMoO}_{4}\end{array}$ & $\begin{array}{l}\text { PANI- } \\
\mathrm{BiMoO}_{4}\end{array}$ & $\begin{array}{l}\text { PANI- } \\
\mathrm{BaMoO}_{4}\end{array}$ \\
\hline 0 & 0 & 0 & 0 & 0 & 0 \\
15 & 1.5 & 1.8 & 15 & 8.2 & 6.4 \\
30 & 1.8 & 2.1 & 22.1 & 15.2 & 10.2 \\
45 & 2.2 & 2.4 & 28.7 & 22 & 18 \\
60 & 2.2 & 2.7 & 35.7 & 26.4 & 22.3 \\
75 & 2.4 & 7.3 & 41.4 & 33.3 & 28.3 \\
90 & 2.4 & 9.8 & 49.2 & 38.2 & 35.6 \\
105 & 2.8 & 11.2 & 54.3 & 44.2 & 40 \\
120 & 2.8 & 15.1 & 58.2 & 50.4 & 44.6 \\
135 & 2.8 & 17.2 & 63.5 & 56 & 47.5 \\
150 & 2.8 & 20.1 & 69.8 & 64.2 & 50.2 \\
165 & 3 & 23.4 & 75.6 & 70.6 & 53.2 \\
180 & 3.1 & 25.2 & 80.2 & 76.1 & 55 \\
\hline
\end{tabular}

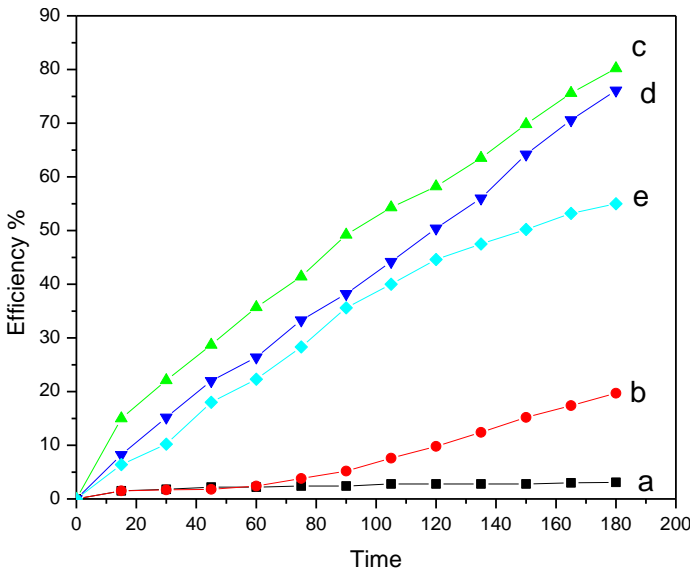

Fig. 5 Photocatalytic efficiency a. Blank; b. PANI; c. PANI-CdMoO 4 ; d. PANI-BiMoO and e. PANI-BaMoO 4

The photocatalytic activity of PANI doped with molybdate enhances because the excited electron generated by $\pi-\pi^{*}$ transition of PANI can be delivered into conduction band of molybdate to the absorbed electron acceptor, thus yielding powerful oxidant (hydroxyl radicals) to degrade $\mathrm{MB}$ dye of water. PANI-CdMoO 4 having higher performance for the degradation of $\mathrm{MB}$ dye under visible lights is due to the following features: the first one is due to have more excellent adsorption capacity than other catalyst compared. Second, PANI-CdMoO ${ }_{4}$ Composite possesses much stronger visible light absorption than bare PANI. Another factor may be due to the synergistic effect between PANI and cadmium molybdate under light irradiation. PANI-C $\mathrm{dMoO}_{4}$ had the maximum activity and hence it is used for further studies.

\section{Conclusion}

The present study presents the synthesis of an electronically and environmentally stable conducting PANI nanocomposite incorporated inorganic nanomaterials of metal molybdates. The polymerisation yields are increased with respect to incorporation of metal molybdates. The growth of polymerization is also enhanced. The incorporation of metal molybdates is confirmed by DC Conductivity, XRD and UV-VIS spectral studies. The XRD studies are also suggested the presence of chemical interaction between polymer chain and incorporated metal molybdates. The anti-microbial activity of four bacterial strain such as Escherichia coli, Staphylococcus aureus, P. aeruginosa and Proteus vulgaris strain using disc diffusion method were evaluated by using four polymeric samples. The nanocomposite doped polymer possesses excellent antibacterial property. The results on MB dye degradation showed that PANI-CdMoO 4 photocatalyst, under visible light degraded $80.2 \%$ of $\mathrm{MB}$ dye after 180 min. The photodegradation study, thus, suggests that the light absorption of PANI and catalytic role of $\mathrm{CdMoO}_{4}$ could be coupled for high photocatalytic functionality. This work opens a new avenue for the design of new modified photocatalysts with high activity for the largescale water treatment and other applications.

\section{Acknowledgement}

Authors are gratefully acknowledged to the UGC for financial assistant in the form of minor research projects UGC-SERO funded minor research projects (MRP-6440/16 dated: 26.03.2017) and (MRP-6844/16 dated: 30.06.2017). Authors are also thankful to the Management and Principal of TDMNS College, T. Kallikulam, Tirunelveli for providing necessary laboratory facilities.

\section{References}

[1] L.F. Johnson, G.D. Boyd, K. Nassau, R.R. Soden, Continuous operation of a solidstate optical maser, Phys. Rev. 126 (1962) 1406-1411.

[2] J.L. Brito, A.L. Barbosa, Effect of phase composition of the oxidic precursor on the HDS activity of the sulfided molybdates of Fe(II), Co(II), and Ni(II), J. Catal. 171 (1997) 467-475.

[3] R. Gangopadhyay, A. De, Conducting polymer nanocomposites: A brief overview, Chem. Mater. 12 (2000) 608-622.

[4] Y. Xian, Y. Hu, F. Liu, Y. Xian, H. Wang, L. Jin, Glucose biosensor based on Au nanoparticles-conductive polyaniline nanocomposite, Biosens. Bioelectron. 21 (2006) 1996-2000.

[5] A.A. Athawale, S.V. Bhagawat, P.P. Katre, Nanocomposite of Pd-polyaniline as a selective methanol sensor, Sens. Actuators B 114 (2006) 263-267. 
[6] W.U. Huynh, J.J. Dittmer, A.P. Alivisatos, Hybrid nanorod-polymer solar cells, Science 295 (2002) 2425-2427.

[7] R.J. Tseng, J. Huang, J. Ouyang, R.B. Kaner, Y. Yang, Polyaniline nanofiber/gold nanoparticle nonvolatile memory, Nano Lett. 5 (2005) 1077-1080.

[8] C.C. Hu, E. Chen, J.Y. Lin, Capacitive and textural characteristics of polyanilineplatinum composite films, Electrochim. Acta 47 (2002) 2741-2749

[9] A. Sen, P. Pramanik, Low-temperature synthesis of nano-sized metal molybdate powders, Mater. Lett. 50 (2001) 287-294.

[10] J.J. Hwang, T.W. Ma, Preparation, morphology, and antibacterial properties of polyacrylonitrile/montmorillonite/silver nanocomposites, Mater. Chem. Phys. $136(2012) 613-623$.

[11] Q. Xiao, J. Zhang, C. Xiao, S. Zhichun, X. Tan, Solar photocatalytic degradation of methylene blue in carbon-doped TiO2 nanoparticles suspension, Solar Energy 82 (2008) 706-713.

[12] R.D. Scipioni, F. Gazzoli, O. Teocoli, A.P. Palumbo, S.B. Nelutalbris, M.A. Navarra Preparation and characterization of nanocomposite polymer membranes containing functionalized $\mathrm{SnO}_{2}$ additives, Membrane 4 (2014) 123-142.

[13] L. Zhang, M. Wang, Polyaniline/TiO 2 composite nanotubes, J. Phys. Chem. B 107 (2003) 6748-6753.

[14] K. Hyevoung, J. Jyongsik, Antibacterial properties of novel poly(methyl methacrylate) nanofiber containing silver nanoparticles, Langmuir 24 (2008) 2051-2056.

[15] T.O. Fufa, A.T. Mengesha, O.P. Yadav, Photocatalytic activity of nanopolyaniline (NPA) on phenol degradation, Afr. J. Pure Appl. Chem. 10 (2016) 33-41.

\section{About the Conference...}

"International Conference on Advanced Materials and Their Applications" has been convened by Dr. C. Vedhi, Assistant Professor, PG and Research Department of Chemistry, V.O. Chidambaram College, Thoothukudi at his designated venue on 27th December 2018. 